\title{
PROCESSING PLATE-PLATE IMMOBILIZATION DATA OF MNFC FURNISHES
}

\author{
Katarina Dimić-Mišić* \\ School of Chemical Technology, Aalto, Finland \\ Kaarlo Nieminen \\ School of Chemical Technology, Aalto, Finland \\ Thaddeus Maloney \\ School of Chemical Technology, Aalto, Finland \\ Herbert Sixta \\ School of Chemical Technology, Aalto, Finland \\ Jouni Paltakari \\ School of Chemical Technology, Aalto, Finland
}

Appropriate regularization algorithm is proposed for choosing the regularization parameter based on nosy immobilization viscosity data. Complex fluids as nanocelluose based high consistency suspensions have very complex rheological behavior. This paper describes Tikhonov regularization procedure for reducing the noise from immobilization rheograms of nanocellulose based furnishes, performed on immobilization cell at MCR 300 rheometer. Noise observed in immobilization rheograms can make evaluation of data very complicated and lead to wrong interpretation of rheological behavior observed. They are related to the change of consistency accompanied with rearrangement of the particles during shear induced dewatering accompanied with wall slip and boundary effects typical for gel like nanocellulose suspensions. Hereby we propose a model with regularization parameters suitable for applying to parallel disk data for flocculated gel like high consistency suspensions while consistency increasing mode upon shearing.

Key words: Data noise, Smoothening, Immobilization, Nanocellulose, Complex suspensions

\section{INTRODUCTION}

Composite materials composed of a mixture of pigments, traditional pulp fibers and cellulose microfibrils (called "nanocellulose") have attracted much attention in the last years 1-3. Such composites can have excellent optical and surface properties and may have an attractive cost structure in the case where a high amount of commercial pigment is used. The rheology and dewatering characteristics of these composite furnishes is very different from both traditional papermaking furnishes, and will therefore require a very different manufacturing paradigm than is common practice today. There is a drive in the paper and board-making industry to produce high consistency furnishes with increased filler content and, thus, to reduce the amount of pulp fibers. Various types of composite materials can be produced from such furnishes if an efficient manufacturing strategy can be developed.
Since such complex suspensions can contain over $20 \%$ of gel -like nanocellulose they have poor dewatering, accompanied with complex thixotropic behaviour resulting in rheograms with significant data noise.

In this article "nanocellulose" refers generically to the material resulting when traditional chemical pulp fibres are defibrillated either by chemical or mechanical means 4. Major diversity is found in raw materials used for the productions of nanofibrillated cellulose and they all possess many special properties compared to traditional pulp fibres; a much higher surface area, great bonding potential, and a high water binding capacity 5,6 .

Microfibrillated cellulose (MFC) can be prepared from chemical pulps by mechanical shearing 7 . If the pulp fibres are oxidized to increase the charge on the cellulose, the fibres can be more easily broken down to a finer distribution of fibrils 
approaching the elementary fibril width of about $4 \mathrm{~nm}$. The TEMPO mediated 8 process for the production of nanofibrillated cellulose (NFC) is currently the focus of research. MFC and NFC represent fairly distinct categories of nanocellulose, each having potential commercial applications. In water dispersions, at concentrations of approximately $1 \%$ solids and higher, nanocellulose forms a highly entangled network consisting of nanoscale size elements with a gel-like behaviour 9,10 . The typical size of NFC is smaller and surface charge much higher compared to MFC. As a result, NFC forms a gel at much lower concentrations. In other words, it binds more water and has higher swelling than MFC. Effect of swelling of fibers on their rheological behaviour has been widely studied 11,12 , and it is apparent that the difference in swelling between MFC and NFC should strongly influence the rheology and dewatering behaviour of furnish.

Both experimental techniques and mathematical models have been developed to describe the water removal in all stages of the paper machine, from the initial forming section through vacuum boxes into press and final thermal water removal 13,14 . Although the composition of papermaking furnishes vary greatly, the main components are the relatively mobile, limitedly swollen fibres, a small amount of more mobile swollen fines, and a variable amount of inorganic non-swelling pigment. It is suggested that plugging of wire with small particles during vacuum dewatering, can be avoided by the judicious application of positive vacuum pulses 1516. The types of nanocelluose based furnishes are high consistency furnishes that contain 70 wt- \% pigment, 20-30 wt- $\%$ nano and/or microfibrillated cellulose and $0-10 \mathrm{wt}-\%$ pulp fibres ( ref cellulose). This is clearly a challenging furnish to dewater, since it contains high amount of fibrillar cellulose material, water (in liquid or vapour form) must pass through relatively small channels around fibrils and pigments. However, if permeability is formed by application of shear which creates new the flow channels within matrix, by the application of mechanical forces, it is possible to improve the dewatering characteristics of furnish (ref cellulose). Shear regime during dewatering imposes stresses on the flow units (particles or flocks which can ) causing the flow units to undergo structural breakdown, leading to viscosity irregularities accompanied with shear thinning and elasticity overshoots which are result of elastic energy within nanocellulose agglomerates 17. Higher more rigorous shear rates break down flow unites that survived exposure to lower shear rates, which is further complicated by continuous solid content increase in dewatering i.e. immobilization measurements 18 . A rheometric resolution for measurement data issue will not be found if the shear stress fails to stabilize in a reasonable length of time19. Rheological measurements are very sensitive to change is consistency, as suspension during shear induced dewatering segregates into solid rich and solid -lean phase which has a net result increase in viscosity, but within different positions of measuring plate20 .The result of two phases coexisting in the annular gap would appear to rheometer as an increase in the apparent average viscosity relative to homogenous sample and in the case of wall slip it results in complicated feedback of rheoneter resulting in high noise of complex and dynamic viscosity data 21-23.

\section{EXPERIMENTAL MATERIALS AND METHODS}

The filler was a commercial undispersed precipitated calcium carbonate with scalenohedral morphology. The particle size was $4.3 \mu \mathrm{m}$ measured with Malvern dynamic light scattering. A commercial bleached pine Kraft bale pulp was refined to a Shopper-Riegler number of 24 with a Valley Beater. MFC was produced from neverdried bleached Birch Kraft pulp. The defibrillation was carried out by passing the pulp three times through a Masuko grinder at $3 w t-\%$ solids 24. The Brookfield (vane) 25viscosity at 10rpm of the MFC was $23000 \mathrm{mPas}$ at $1.5 \mathrm{wt}$ - $\%$ solids. The measured zeta potential was $-24.3 \mathrm{mV}$ at $\mathrm{pH}$ 9.5. The transmittance for $0.1 \mathrm{wt}-\%$ consistency for the wavelength of $800 \mathrm{~nm}$ was $34 \mathrm{wt} \% 26$. The applied NFC sample was a semi-commercial one, produced with the TEMPO process 27 from the never dried birch pulp. The Brookfield (vane) viscosity of the NFC at $1.5 \mathrm{wt}-\%$ solids at $10 \mathrm{rpm}$ was $46657 \mathrm{mPas}$. The measured zeta potential was $-57 \mathrm{mV}$ at $\mathrm{pH}$ 9.2. Furnishes were made for three consistencies 5, 10 and 15 wt- $\%$ solids content. The furnish composition was 70 wt- $\%$ filler, $20-30$ wt- $\%$ MFC or NFC, and 0-10 wt- $\%$ cellulose fibres. All the labels follow the notation: ratio of pigment / ratio and type of nanocellulose wt- $\%$ / ratio of pulp wt- $\%$ / consistency wt- $\%$. The MFC or NFC was mixed with filler at vigorous rotation with laboratory Diaf mixer for 
25 minutes and the cellulose pulp was added to this suspension with further mixing at the same mixer as above. De-ionized water was used for dissolution and preparation throughout the study. The samples were stored in cold at $5^{\circ} \mathrm{C}$ temperature. The swelling of MFC and NFC was evaluated with a modified water retention test WRV ISO/DIS 23714.

\section{RHEOLOGICAL MEASUREMENTS}

Physica MCR-300 rheometer was used in controlled shear mode with plate-plate geometry28, 29. The rheometer was equipped with $25 \mathrm{~mm}$ plate-plate geometries with both upper and lower surface profiled (serrated) with a $0.5 \mathrm{~mm}$ deep texture30. The gap was initially set to $1 \mathrm{~mm}$ and the sample was allowed to rest for $10 \mathrm{~min}$ before starting the measurement. The temperature was set to a constant value of $23^{\circ} \mathrm{C}$. Both MFC suspensions and MFC furnishes were measured applying a strain sweep, frequency sweep, direct strain-controlled oscillatory (DSO) mode, and shear flow in CSR mode. In the strain sweep the strain was varied between 0.1 to $500 \%$ and the angular frequency was set to $1 \mathrm{~Hz}$. Next, the frequency sweep was conducted after determining the linear viscoelastic region, using the amplitude sweep at constant strain of $y=0.1 \%$ with varying angular frequency from 100 to $0.01 \mathrm{rad} \mathrm{s}^{-1}$.

\section{IMC-IMMOBILIZATION CELL DEWATERING}

Dynamic dewatering measurement was conducted using a Physica MCR 300 rheometer with IMC accessory as licensed from BASF-AG. The cell consists of a stainless steel cylindrical metal tube with a punched metal plate that allows drainage of water through the samples 29, 31. A filter is fastened on the top of the metal plate and the furnish to be investigated is applied. The upper plate is then lowered into contact with sample which is then pre-sheared. The sample is dewatered through the filter by vacuum suction. The normal forces of the sample tend to push upper rotating plate. The axial force acting on the upper plate is set to a constant value, as described by other authors 28 .A parallel plate geometry measuring system (PP-50) with a $50 \mathrm{~mm}$ diameter was used in all immobilization tests and initial gap was set to $1 \mathrm{~mm} 29$. using a Whatman nuclepore membrane, with the pore size of 0.2 $\mu m$. For the purpose of this study we modified a traditional IMC measurement used for coating colour dewatering 29, 32, 33 . However, in the case of complex structures which are undergoing change, such as those studied in this work, the linear viscoelastic (LVE) assumption will likely not apply across the transitions between the various structural states involved18. Nonetheless, within each structural state, i.e. free flowing through localized gel formation to immobilization, it can be reasonably assumed that the behavior is individually acting in its own LVE state. Thus, following the rates of change of the viscoelastic parameters, namely in the form of the rate of change of the loss factor, as proposed in this study, duly identifies the structural change points as a function of solids content34. Vacuum filtration induces a gradient in the solid content in the filter normal direction 35, thus increasing inhomogeneities within the sample, and therefore the rheological properties of the samples measured in this way correspond to their apparent values specific for this geometry and can be considered suitable only for comparative studies.

Two testing approaches can be applied with the IMC: the controlled shear rate (CSR) and the direct strain-controlled oscillatory (DSO) method. The tan $\delta$, which is the ratio of $G^{\prime \prime} / G^{\prime}$, marks the transition of liquid like to solid-like behavior during dewatering 29 . The DSO procedure thus provides dynamic rheological information for a viscoelastic material during dewatering in the IMC through change of the complex viscosity $\left(n^{*}\right) 36$. Traditionally, two specific points are defined to characterize the immobilization of coating colors 29 , and we believe that they can essentially be used to characterize suspensions in general.

Parameters for DSO measurement were within LVE. However, during the CSR tests, the viscoelastic properties could not be determined, instead only the change in dynamic viscosity $\eta$ could be monitored 37. In our dewatering models, DSO was used with the application of vacuum. CSR intervals were applied between the DSO intervals with shear rate of $y=0 / 40 / 200 \mathrm{~s}^{-1}$. Dewatering with vacuum of 50 mbar and the duration of 400 $s$ was performed during the DSO intervals, due to the fact that applying vacuum during the CSR cycle would make the small particles to plug the filter pores. The samples were pre-sheared in the DSO cycle for $40 \mathrm{~s}$, in the first interval, and then the periodic succession of DSO and CSR proceeded. During with CSR intervals the vacuum pump was switched off manually with the duration of these intervals being $10 \mathrm{~s}$. 
Shear rate was limited due to the viscoelastic Weissenberg response typical for polymers as nanocellulose is, which results in sample to concentrate towards the centre of the bottom plate tending to increase the upper measuring plate adding to inhomogeneity within the sample (cellulose). Additionally dewatering in only DSO mode was performed with duration of $t=400 \mathrm{~s}$ and increase of $\eta^{*}$ monitored.

\section{TIKHONOV REGULARIZATION}

Regularisation algorithms are often used to produce reasonable solutions to ill-imposed problems by first unifying characterization of various regularisation methods and the by observing final smoothed data particular regularization method is chosen $20,38,39$.

\section{Results}

Tikhonov regularization 40 is a renowned method for solving ill-posed problems of type:

$$
A \vec{x}=\vec{y}
$$

where A is a matrix and $\vec{x}, \vec{y}$ vectors. In equation (1) $\vec{y}$ represents an observed variable, the measurements of which are subjected to some noise whereas $\vec{x}$ on the other hand is an unmeasured quantity. Further the linear relationship between $\vec{x}$ and $\vec{y}$ is described by the matrix $\mathrm{A}$. To calculate $\vec{x}$ from $\vec{y}$ some kind of inversion of the matrix A must be employed, hence the problem is also related to as an inverse one. With the problem being ill-posed it is meant that no unique solution exists or that the solution is very sensitive even to small fluctuations in $\vec{y}$. In Tikhonov regularization the minimization problem:

$$
\min _{x}\|A \vec{x}-\vec{y}\|^{2}
$$

is replaced by the penalized minimization problem:

$$
\min _{x}\left\{\mid A \vec{x}-\vec{y}\left\|^{2}+\lambda\right\| L \vec{x} \|^{2}\right\}
$$

where $L$ is a matrix denoted the regularization matrix and $\lambda$ is a positive parameter.

The purpose of the second term in the expression to be minimized is to penalize extreme solutions and favor physical ones. If e.g. the identity matrix is selected as regularization matrix solutions with small norm will be favored. The appropriate value for the smoothing parameter $\lambda$ depends on the measurement frequency and the amount of noise in the data. In most cases of this study the measurement frequency was 0.5 $\mathrm{Hz}$. Then the initially attempted value for the $\lambda$ parameter was 6400 . In some noisy cases the value was increased up to 100000 to obtain a smooth function.

In this study Tikhonov regularization is applied, not for calculating some unmeasured quantities from measured ones, but as tool for smoothening measured data. This is achieved by setting the matrix A in Equation (3) to the identity matrix and selecting the regularization matrix in such a way that it penalizes data fluctuations. With the Tikhonov regularization based smoothing method we obtained proper rheograms for high consistency micro- and nanocellulose suspensions also in the critical segments, where solid content increases and phase separation starts.

Rheological data involving MFC and NFC, contained high-frequency mechanical noise, which, unless stated otherwise, was subsequently smoothed by modified Tikhonov regularization 39-41. Solutions with smaller curvatures were preferred by setting the forward operator to the identity matrix and the regularization operator to a discretized form of the second derivative. The balance between fitting the original noisy data and smoothness was adjusted by a scaling parameter. Immobilization descriptors were calculated from five successive measurements. For the plotted rheograms in the following results, data from representative measurements only are used to exemplify the behavior.

Suppose we have a vector consisting of noisy measurements at equal intervals in time $\Delta t$. We want to replace it by another vector that is smoother, but still approximates the original vector reasonably well. To achieve this goal we seek for the vector that minimizes the sum:

$\|\vec{u}-\vec{v}\|^{2}+\lambda\|L \vec{u}\|^{2}$

where, as in equation ( 3 ), $\lambda$ is a positive constant and $L$ is an operator (in this context a matrix) that estimates the amount of random noise in the vector it acts on. The first term in the expression measures the distance of the new vector from the original one, the second the unsmoothness of the new vector. The choice of the constant $\lambda$ depends on how much we want to emphasize either the original data or the smoothness. If $\lambda=0$, then (4) is minimized by the original data, 
if $\lambda \rightarrow \infty$,the components of the minimizing vector as a function of time approaches a straight line. The second derivative, which gives a local estimate of the curvature of a function, was se- lected as operator (matrix) L. With the standard finite difference approximation of the second derivative this matrix becomes:

$$
L=\left(\begin{array}{ccccccccccc}
1 & -2 & 1 & 0 & 0 & & & & & \\
0 & 1 & -2 & 1 & 0 & \cdots & & & 0 & & \\
0 & 0 & 1 & -2 & 1 & & & & & \\
& & \vdots & & & \ddots & & & \vdots & & \\
& & 0 & & & \ldots & 1 & -2 & 1 & 0 \\
& & & & & & 0 & 1 & -2 & 1
\end{array}\right) \frac{1}{(\Delta t)^{2}}
$$

To minimize the expression (4) we demand that all its partial derivatives with regard to the components of $\vec{u}$ are zero. In order to be able to evaluate the derivatives we write the first term in the expression (4) as:

$$
\|\vec{u}-\vec{v}\|^{2}=(\vec{u}-\vec{v})^{T}(\vec{u}-\vec{v})=\sum_{i}\left(u_{i}-v_{i}\right)^{2}
$$

and the second term as:

$$
\begin{aligned}
& \lambda\|L \vec{u}\|^{2}=\lambda \vec{u}^{T} L^{T} L \vec{u}=\lambda \vec{u}^{T} B \vec{u}=\lambda \sum_{i, j} u_{i} b_{i, j} u_{j} \\
& \partial_{k} \lambda \sum_{i, j} u_{i} b_{i, j} u_{j}=\lambda \sum_{i, j}\left(b_{i, j} u_{j} \partial_{k} u_{i}+b_{i, j} u_{i} \partial_{k} u_{j}\right)=\lambda \sum_{j} b_{k, j} u_{j}+\lambda \sum_{i} b_{i, k} u_{i}=2 \lambda \sum_{j} b_{k, j} u_{j}
\end{aligned}
$$

The last step in Eq.9 follows from the symmetry property of the matrix $B$. In vector form the zero condition of the partial derivatives is:

$\vec{u}-\vec{v}+\lambda L^{T} L \vec{u}=0$

The vector $\vec{u}$ minimizing the expression (4) is then obtained as the solution of the equation:

$$
\left(I+\lambda L^{T} L\right) \vec{u}=\vec{v} .
$$

Here $l$ is the identity matrix. The $\sim$ lution $\vec{u}$ is the smoothed version of the vector $\vec{v}$.

\section{IMMOBILIZATION MEASUREMENTS}

At Figure 1 is presented NFC furnish with $10 \%$ of cellulose fibers, $20 \mathrm{wt}-\%$ of NFC and $10 \mathrm{wt} \%$ of pulp fibers, in DSO dewatering mode. Plot is showing increase in $\eta^{*}$ after application of vacuum due to increase of solid content. Significant data noise is observable after startup of immobilization, and progressing as increase in $\eta^{*}$ slows down and slowly reaching its plateau, which is very difficult to determine due to highly gellike behavior of NFC 42. With increase in time, as the solids content of sample increases data noise also increases due to the differentiation within the samples induced by the solids gradient within measuring geometry accompanied with wall slip effect. After applying smoothening algorithm proposed above, we can see smooth rheogram upward curve following initial increase in solid content immobilization 28, 29, 37.

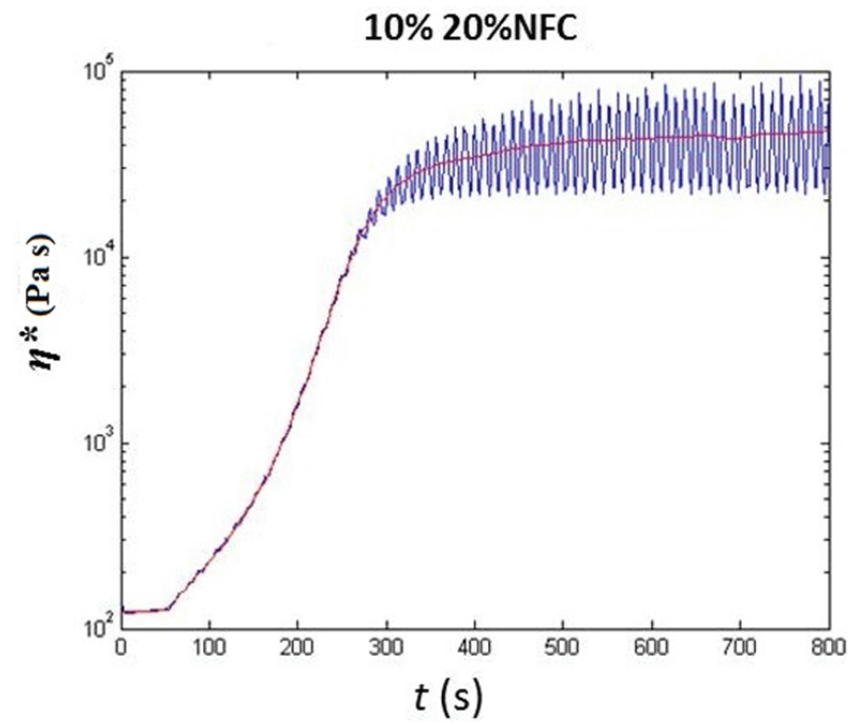

Figure 1: Immobilization as an increase of complex viscosity $\eta^{*}$, noise data and smoothed data 
Figure 2 shows a $\eta^{*}$ rheogram for 10 wt- $\%$ NFC furnish and 30 wt- $\%$ of nanocellulose in combined DSO and CSR dewatering. Figure presents increase of the solids content through increase of the $\eta^{*}$ (DSO) 29, 32. Apart from the first (pre-shearing) DSO interval, we see that $\eta^{*}$ in each following DSO interval is dependent on the viscoelastic response of furnish during the previous CSR intervals18. During DSO interval $\eta^{*}$ has a non-linear dependency on solids content of the furnish cake, shear thinning during the DSO interval, shearing history in previous CSR intervals, and the Weissenberg effect.

As in the previous figure we see data noise with increase in solid content accompanied with increase in complex viscosity, which is more observable after first two cycles of CRS, and with progressing of dewatering decreases, as the structure stabilizes, complex viscosity noise is higher immediately after shear thinning in CSR cycle, as the structure is broken during shearing which results in irregularities within the shear profile within the thixotropic sample 43. An increase in the $\eta^{*}$ during DSO and dewatering $t$, is followed by a profound shear thinning during CSR intervals, seen as very low $\eta^{*}$ at the beginning of DSO cycle10, 21 .This suggests that a potentially complicated relationship exists between rheology, solids content and suspension concentration 44.

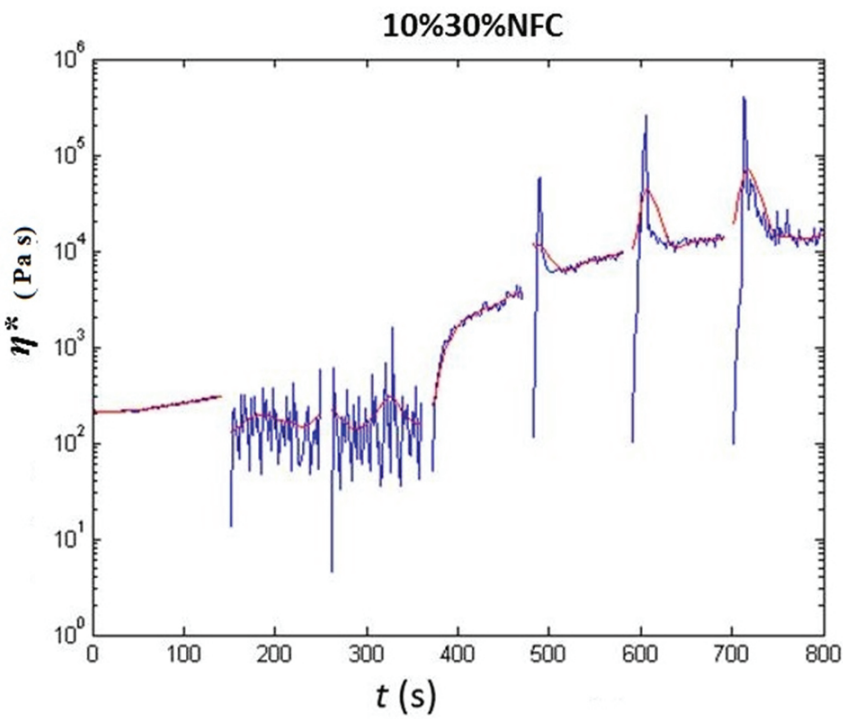

Figure 2: Development of complex viscosity $\eta^{*}$ in DSO cycles with vacuum dewatering after the rotation CSR cycles with shear rate of $100 \mathrm{~s}^{-1}$

Data noise is more pronounced after application of shear $\left(\gamma=0 / 40 / 200 \mathrm{~s}^{-1}\right)$, as presented in Figs. $3 a)-b)$. In the first stage of the dewatering pro- cess, the rheological behavior is determined to a large extent by the sample viscoelasticity. Data presented in Fig. 3 a) show a continuous increase of G' for MNFC furnishes (can be understood as increase of elastic part and decrease of loss factor ( $\tan \delta$ )34 up to its maximum before the $\eta^{*}$ reaches its plateau seen in Fig.3b). The very slow evolution of $G^{\prime}$ and $\eta^{*}$ in NFC furnishes is already discussed, and relates, in addition to the lack of aggregation due to higher swelling, also to the partial sealing of the bottom layer by the highly swollen fibrils, which prevents dewatering to complete interlocking immobilization, resulting in a situation of effectively trapped/bound water in the matrix. However, since there is no rapid increase shown in the complex viscosity right after the on-set of vacuum, we expect that slower dewatering rate of NFC is primarily due to higher swelling. MFC furnishes have very deifferent viscoelastic response to the dewatering process than NFC furnishes The highly swollen NFC makes water removal difficult due to the sealed bottom layer of the filter cake, making dewatering and immobilization slow. Therefore, $n^{*}$ and $G^{\prime}$ of NFC furnishes have a maximum which develops much later than in the case of MFC suspensions and furnishes. After DSO cycles there are observed irregularities in both $\eta^{*}$ and $G^{\prime}$ in DSO cycles, which are higher for higher shear rate applied. This irregularities are not seen for the zero shear rate, indicating that with shear INC dewatering different phase separation effects occur. Also noticeable is lower $\eta$ for CSR with higher shear rates.

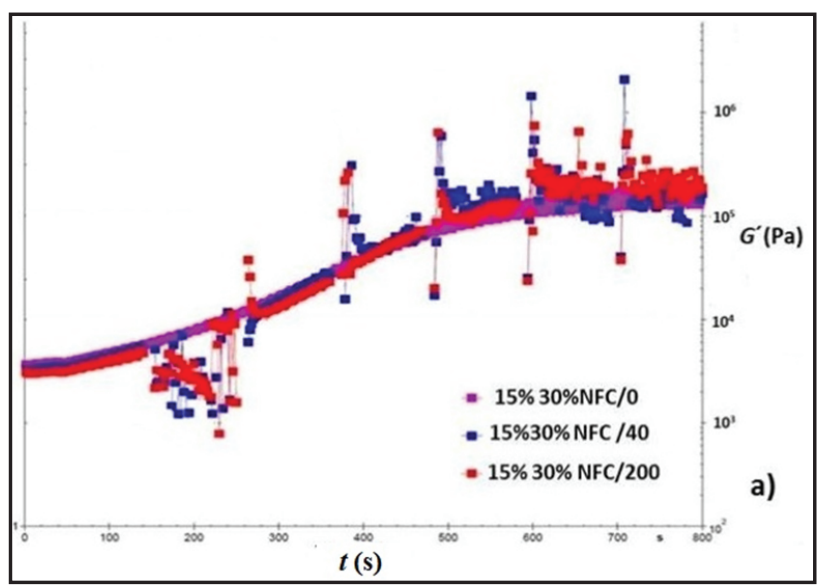

Figure 3: Apparent variation noise of rheological data at shear rate $\dot{\gamma}=0 / 40 / 200 \mathrm{~s}^{-1}$ CSR dewatering for a) $G^{\prime}$ for 15 wt-\% MNFC furnishes 


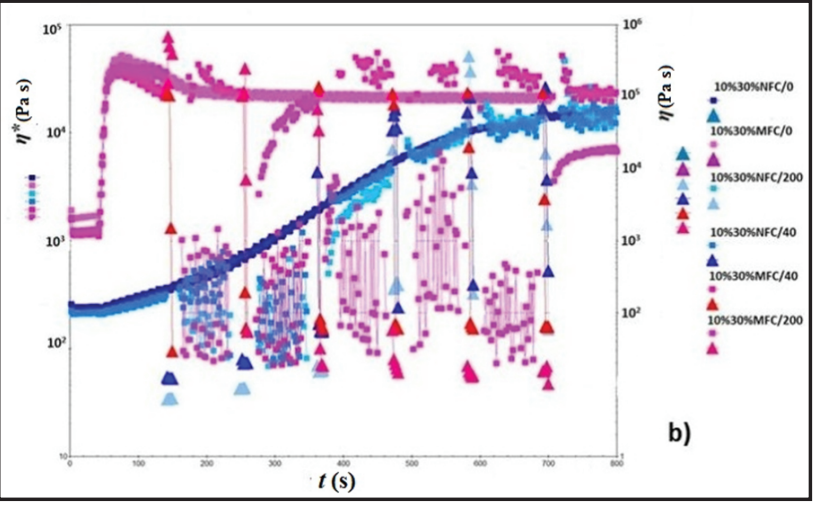

Figure 3: Apparent variation noise of rheological data at shear rate $\dot{\gamma}=0 / 40 / 200 \mathrm{~s}^{-1} \mathrm{CSR}$ dewatering for $b) \eta^{*}$ and $\eta$ for the $10 w t-\%$ MNFC furnishes with data structure response variation immediately after application of vacuum

After applying the smoothing algorithm for $\eta^{*}$ in the case of both MFC and NFC furnishes, we see in Figure 4 that the increase in consistency during immobilisation, under the applied DSO mode, is dependent upon nanocellulose origin. The relative structure properties then relate to aggregation, flocculation (in case of MFC) and gel formation with trapped water (NFC) defined by the gel swelling degree in respect to adsorbed and interstitial water within the nanocellulose matrix.

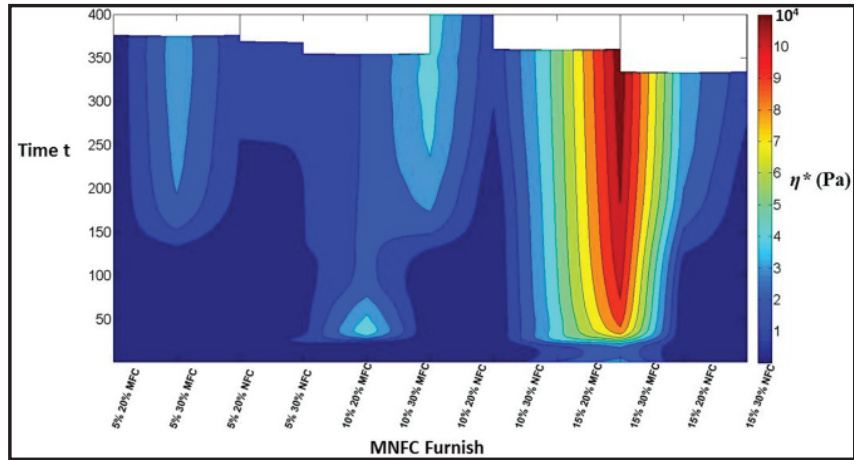

Figure 4: Increase in complex viscosity $\left(\eta^{*}\right)$ as smoothed data rheogram for 5-15 wt- \% consistency MNFC-containing furnishes in DSO mode

Application of smoothing algorithm for $\eta^{*}$ in DSO dewatering mode of MNFC furnishes, presented Figure 4 gives nicely indication of increase in consistency during immobilisation, being dependent dependent upon nanocellulose origin as for flocculated MFC phase separation is helping dewatering, while for gel like NFC increase in consistency is much more reduced due to watter traped in gel-like matrix. Those esults will be impossible to obtain with raw data and therefore we emphasize importance of using this algorithm when dealing with change in solid measurements on the plate-plate geometry.

\section{CONCLUSION}

The IMC enables the application of vacuum dewatering under shear by using smooth plateplate geometry. This is a significant advantage when trying to understand the application of shear as a means to improve the dewatering of the MFC/NFC furnishes. Immobilization cell is designed as plate-plate geometry device and as such is prone to shear distribution irregularities upon shearing and dewatering high consistency MNFC furnishes. The very high elasticity of the MNFC furnishes results in the complex behaviour under dewatering conditions, uneven distribution of shear, wall slip, and the Weissenberg effect, typically accompanying parallel-disk geometries further complicated by solid content gradient. We have proposed a model developed specially for this type of data fluctuations based on Tikhonov regularization which is suitable for this type of suspensions at plate-plate geometry and demonstrated procedure by applying it to the high noise data.

\section{REFERENCES}

1) Agoda-Tandjawa, G.; Durand, S.; Gaillard, C.; Garnier, C.; Doublier, J. -. Carbohydr. Polym. 2012, 2, 1045-1057.

2) Ayol, A.; Dentel, S. K.; Filibeli, A. J. Environ. Eng. 2010, 9, 992-999.

3) Barnes, H.; Carnali, J. J. Rheol. 1990, 841.

4) Bertola, V.; Bertrand, F.; Tabuteau, H.; Bonn, D.; Coussot, P. J. Rheol. 2003, 1211.

5) Chang, G.; Koo, J.; Song, K. Korea-Australia Rheology Journal 2003, 2, 55-61.

6) Chinga-Carrasco, G.; Kuznetsova, N.; Garaeva, M.; Leirset, I.; Galiullina, G.; Kostochko, A.; Syverud, K. Journal of Nanoparticle Research 2012, 12, 1280.

7) Dimić, K.; Maloney, T.; Paltakari, J.; Sanavane, Y. Journal of Applied Engineering Science (Istrazivanja i projektovanja za privredu) 2013, 3,

8) Dimić-Mišić, K.; Paltakari, J. Journal of Applied Engineering Science 2012, 4, 209220.

9) Dimic-Misic, K.; Puisto, A.; Gane, P.; Nieminen, K.; Alava, M.; Paltakari, J.; Maloney, T. Cellulose 2013, 1-15. 
10) Dimic-Misic, K.; Puisto, A.; Paltakari, J.; Alava, M.; Maloney, T. Cellulose , 2012, 20, 4.

11) Dimic-Misic.K.,Gane,P.A.C.,Paltakari J., Micro-and Nanofibrillated cellulose as rheology modifier additive in CMC-containing pigment coating formulations ndustrial and Engineering Chemistry Research Journal2013, 52 (45)161600-16083

12) Divoux, T.; Grenard, V.; Manneville, S. Phys. Rev. Lett. 2013, 1, 018304.

13) Hansen, P. C.; O'Leary, D. P. SIAM Journal on Scientific Computing 1993, 6, 1487-1503.

14) Henriksson, M.; Henriksson, G.; Berglund, L.; Lindström, T. European Polymer Journal 2007, 8, 3434-3441.

15) Horvath, A. E.; Lindström, T. J. Colloid Interface Sci. 2007, 2, 511-517.

16) Hubbe, M. A.; Heitmann, J. A. BioResources 2007, 3, 500-533.

17) Hubbe, M. A.; Panczyk, M. O Papel (Brazil) 2007, 10, 88-100.

18) Hubbe, M. A.; Rojas, O. J.; Lucia, L. A.; Sain, M. BioResources 2008, 3, 929-980.

19) lotti, M.; Gregersen, $\varnothing$ W.; Moe, S.; Lenes, M. Journal of Polymers and the Environment 2011, 1, 137-145.

20) Jader, J.; Jarnstrom, L. Applied Rheology 2003, 3, 125-131.

21) Jäder, J.; Järnström, L.; Engström, G. Nordic Pulp and Paper Research Journal 2003, 4, 382-387.

22) Karppinen, A.; Vesterinen, A. H.; Saarinen, T.; Pietikäinen, P.; Seppälä, J. Cellulose 2011, 1381-1390.

23) Laine, J. In In Fibre surface modification by different polyelectrolytes - A possibility to obtain new paper properties; PulPaper 2007 Conference: Innovative and Sustainable use of Forest Resources; 2007;

24) Landman, K.; Sirakoff, C.; White, L. Physics of Fluids A: Fluid Dynamics 1991, 1495.

25) Lasseuguette, E.; Roux, D.; Nishiyama, Y. Cellulose 2008, 3, 425-433.

26) Lindström, T.; Carlsson, G. Svensk Papperstindning-Nordisk Cellulosa 1982, 3, 14-20.

27) Mezger, T. G. The rheology handbook: for users of rotational and oscillatory rheometers; Vincentz Network GmbH \& Co KG: 2006;

28) Møller, P. C. F.; Mewis, J.; Bonn, D. Soft Matter 2006, 4, 274-283.
29) Nakagaito, A. N.; Fujimura, A.; Sakai, T.; Hama, Y.; Yano, H. Composites Sci. Technol. 2009, 7, 1293-1297.

30) Pajari, H.; Koskela, H. In In 9.2 - Consolidation of coating colors - Experimental studies; 11th Advanced Coating Fundamentals Symposium Proceedings: The Latest Advances in Coating Research and Development; 2010; pp 347-359.

31) Paradis, M. A.; Genco, J. M.; Bousfield, D. W.; Hassler, J. C.; Wildfong, V. Tappi J. 2002, 8, 12-18.

32) Press, W. H.; Flannery, B. P.; Teukolsky, S. A.; Vetterling, W. T. Numerical Recipes in FORTRAN 77: Volume 1, Volume 1 of Fortran Numerical Recipes: The Art of Scientific Computing; Cambridge university press: 1992; Vol. 1.

33) Puisto, A.; Illa, X.; Mohtaschemi, M.; Alava, M. Nordic Pulp and Paper Research Journal 2012, 2, 277.

34) Puisto, A.; Illa, X.; Mohtaschemi, M.; Alava, M. The European Physical Journal E 2012, 1, 1-7.

35) Pujara, J.; Siddiqui, M.; Liu, Z.; Bjegovic, P.; Takagaki, S.; Li, P.; Ramaswamy, S. Drying Technol 2008, 3, 341-348.

36) Saito, T.; Isogai, A. In In TEMPO-mediated oxidation of native cellulose; Appita Annual Conference; 2005; Vol. 3, pp 337-340.

37) Siró, I.; Plackett, D. Cellulose 2010, 3, 459-494

38) Subramanian, R.; Hiltunen, E.; Gane, P. A. C. Cellulose Fibers: Bio-and Nano-Polymer Composites: Green Chemistry and Technology 2011, 121.

39) Turbak, A. F.; Snyder, F. W.; Sandberg, K. R. In In Microfibrillated Cellulose-A New Composition of Commercial Significance; TAPPI Press: Atlanta, Ga., USA, 1984; pp 115-124.

40) Willenbacher, N. Current Opinion in Colloid and Interface Science 2011, 1, 1-2.

41) Wollny, K. Applied Rheology 2001, 4, 197-202.

42) Yeow, Y. L.; Chandra, D.; Sardjono, A. A.; Wijaya, H.; Leong, Y.; Khan, A. Rheologica acta 2005, 3, 270-277.

43) Yeow, Y. L.; Leong, Y.; Khan, A. Applied Rheology 2007, 6, 66415-66493.

44) Тихонов, A In In О решении некорректно поставленных задач и методе регуляризации; Докл. АН CCCP; 1963; Vol. 151, pp 501-504.

Paper sent to revision: 29.11.2013.

Paper ready for publication: 09.06.2014. 